Proceedings of the International School and Conference on Optics and Optical Materials, ISCOM07, Belgrade, Serbia, September 3-7, 2007

\title{
Proposed Experiment with Rydberg Atoms to Study the Influence of Particle Size on Quantum Interference
}

\author{
M. Gondran ${ }^{a}$, M. BožIĆc ${ }^{b, *}$, D. Arsenovićc ${ }^{b}$ And A. Gondran ${ }^{c}$ \\ ${ }^{a}$ EDF, Research and Development, Clamart, France \\ ${ }^{b}$ Institute of Physics, Pregrevica 118, P.O. Box 68, 11080 Belgrade, Serbia \\ ${ }^{c}$ SET, Université Technologique de Belfort, Montbeliard, France \\ We are looking for the answers to some questions which naturally arise \\ when one starts to think about possible influence of the size of a particle on \\ the transmission, diffraction and interference pattern behind an interference \\ grating. \\ PACS numbers: 03.75.Dg, 07.60.Ly, 03.65.Ta
}

\section{Introduction}

Atomic interferometry demonstrates quantum properties of atomic center of mass motion. In a class of atomic interference experiments internal states do not change, so that it is sufficient to consider only external states in explaining these experiments. But, in a wider class of atomic interference experiments internal states change and play an important role. A quite old experiment of Haroche's team [1] with the Rydberg atoms shows that the Rydberg atoms do not pass through $1 \mu \mathrm{m}$ wide slits if their principal quantum number is rather large $(n>60)$. Thus, the particle density measured after the slits are null. What about a wave function behind the array of narrow slits?

The wave function of an incident atom is the product of a wave function of the center of mass motion and of an internal wave function. What is the influence of slits upon each one of them? Whether both functions are equal to zero behind the slits?

In the traditional explanation of this experiment the latter two questions have not been posed. The standard interpretation considers only a wave function of the center of mass motion and supposes that if the particles do not pass, a wave

*corresponding author; e-mail: bozic@phy.bg.ac.yu 
function of a particle, implicitly identified with a wave function of the center of mass, is null behind the slits (standard assumption).

But, an internal wave function describes the size of a particle. Therefore, logically one should expect that the slits influence an internal wave function. For particle size larger than a slit, one may assume that this function reduces to zero. Consequently, one cannot thus eliminate logically alternative interpretation of this experiment, which supposes that the wave function of external motion passes through the slits, but not the particle (alternative assumption) $[2,3]$.

In this paper we propose an experiment testing this alternative assumption compared to the standard assumption. It is an experiment with large particles (Rydberg atoms or large molecules) sent through a grating consisting of: one wide slit which lets the particles pass, and many small slits which do not let them pass. Theoretical predictions under the two different assumptions, mentioned above, are derived using numerical simulation of particles' arrivals to a screen after passing through this complex grating. This numerical simulation is based on the time dependent solution of Schrödinger's equation for a quantum particle behind a grating $[3-5]$.

\section{The Rydberg atoms asymmetric experiment}

An asymmetric Rydberg atoms experiment, inspired by the Fabre et al. experiment [1], is proposed. A Rydberg atoms' beam with a principal quantum number $n=60$, whose speed is $v=200 \mathrm{~m} / \mathrm{s}$ along the $y$-axis, is considered. Initial speeds in the other directions are considered null. The beam is regarded as plane wave.

At the distance $d_{1}$ from the molecular beam source, a complex grating is placed consisting of the $100 \mu \mathrm{m}$ wide slit A along the $x$-axis and a Ronchi grating $\mathrm{B}$, with 1000 small slits of $0.1 \mu \mathrm{m}$ width. The distance between the centers of slit A and grating B is $300 \mu \mathrm{m}$. Rydberg atoms are then observed by using a detector placed at $y=2 \mathrm{~m}$ behind the slits. The Rydberg atom beam is obtained from sodium atoms, so the atom mass is $m=3.84 \times 10^{-26} \mathrm{~kg}$. With the velocity $v=200 \mathrm{~m} / \mathrm{s}$, the de Broglie wavelength $\lambda=2 \pi / k=8.6 \times 10^{-5} \mu \mathrm{m}$ is much smaller than the slits size.

The slit size of the Ronchi grid B $(0.1 \mu \mathrm{m})$ is ten times smaller than for the Fabre et al. experiment [1]. In this case, the Rydberg atoms with $n=60$ do not pass through the grid. On the other hand, slit A is 100 times larger than the Rydberg atoms' size and thus lets them pass easily.

For $n=60$, the $70 \mathrm{~ms}$ of the Rydberg atom lifetime are sufficient for the experiment (15 ms).

The case of the classical assumption where the wave function does not pass through the grid B corresponds to diffraction by the slit A. The case of the alternative assumption where the wave function passes through the grid $\mathrm{B}$ and slit $\mathrm{A}$ corresponds to an interference problem between the slit A and the grid B slits. 


\section{Wave function calculation with Feynman path integral method, Fresnel-Kirchhoff method and transverse momentum distribution method}

The wave function of the center of mass behind a grating may be evaluated using Feynman path integrals [6], as Gondran and Gondran [5] did for the numerical simulation of Shimizu et al. experiment with cold atoms. With an incident wave function falling to a grating $\psi^{\mathrm{i}}\left(x, 0^{-}\right)$is associated the time-dependent wave function $\psi(x, t)$ :

$$
\psi(x, t)=\int_{A / B} K\left(x, t ; x^{\prime}, 0^{-}\right) \psi^{\mathrm{i}}\left(x^{\prime}, 0^{-}\right) \mathrm{d} x^{\prime},
$$

where $K\left(x, t ; x^{\prime}, 0\right)$ is the free particle propagator given by

$$
K\left(x, t ; x^{\prime}, 0\right)=\left(\frac{m}{2 \mathrm{i} \pi \hbar}\right)^{1 / 2} \exp \left(\frac{\mathrm{i} m}{\hbar} \frac{\left(x-x^{\prime}\right)^{2}}{2 t}\right) .
$$

The integration in (1) is carried out respectively on the slit A and on the grid B slits. The same expression for the wave function is derived using the FresnelKirchhoff integral [4] under the assumption that a motion along $y$ axis is classical, satisfying the relation $y=v t$. It was shown by Arsenovic et al. [7] that the form (1) is equivalent to the following integral over transverse momentum of a particle:

$$
\psi(x, t)=\frac{1}{\sqrt{2 \pi}} \int_{-\infty}^{\infty} \mathrm{d} k_{x} c\left(k_{x}\right) \mathrm{e}^{\mathrm{i} k_{x} x} \mathrm{e}^{-\mathrm{i} \hbar k_{x}^{2} t / 2 m},
$$

where $c\left(k_{x}\right)$ is the Fourier transform of the initial wave function

$$
c\left(k_{x}\right)=\frac{1}{\sqrt{2 \pi}} \int_{-\infty}^{\infty} \mathrm{d} x \psi\left(x, 0^{+}\right) \mathrm{e}^{-\mathrm{i} k_{x} x} .
$$

The function $\psi\left(x, 0^{+}\right)$is equal to the incident wave function for points $x$ at the slits and is equal to zero for points $x$ in between the slits. The function $c\left(k_{x}\right)$ is called probability amplitude of particle transverse momentum, or the transverse wave function in momentum representation.

In the far field the wave function is approximated by [4]:

$$
\psi(x, t)=\sqrt{\frac{m}{\hbar t}} \mathrm{e}^{-\mathrm{i} \pi / 4} \mathrm{e}^{\mathrm{i} x^{2} m / 2 \hbar t} c\left(x \frac{m}{\hbar t}\right) .
$$

Far from the slits, particle distribution in the far field is proportional to the momentum distribution, where $x \frac{m}{\hbar t}$ plays the role of $k_{x}$. By evaluating the quantum mechanical current associated with wave function (5) one concludes that particle trajectories in the far field are straight lines characterized by the ratio of a transverse momentum over the momentum along $y$ axis.

For a grating described in Sect. 2, the probability density of transverse momentum $\left(p_{x}=\hbar k_{x}\right)$ is represented in Fig. 1. Central maximum is the sum of the central maximum corresponding to the wide slit $\mathrm{A}$ and of the central maximum corresponding to the Ronchi grating B. Two small maxima correspond to the Ronchi grating. 


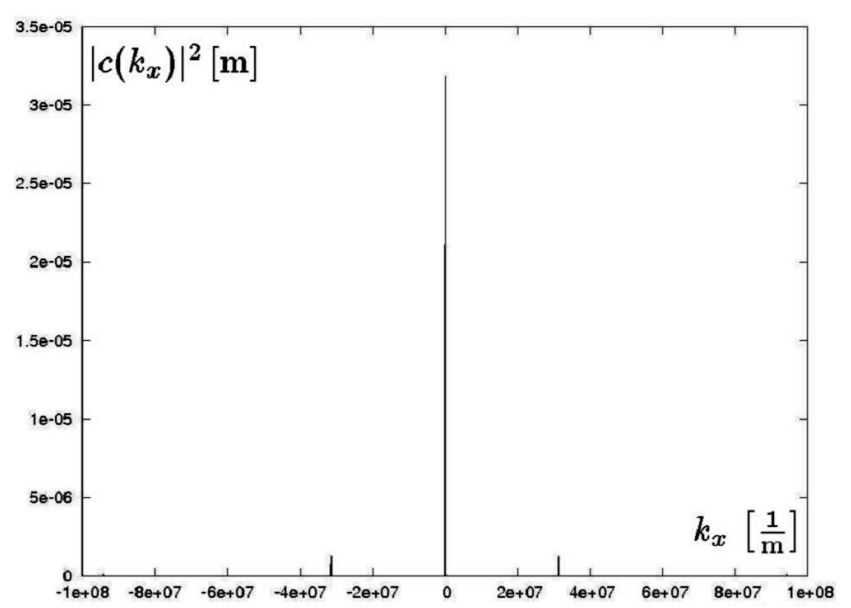

Fig. 1. Probability density of transverse momentum for a complex grating described in Sect. 2 .

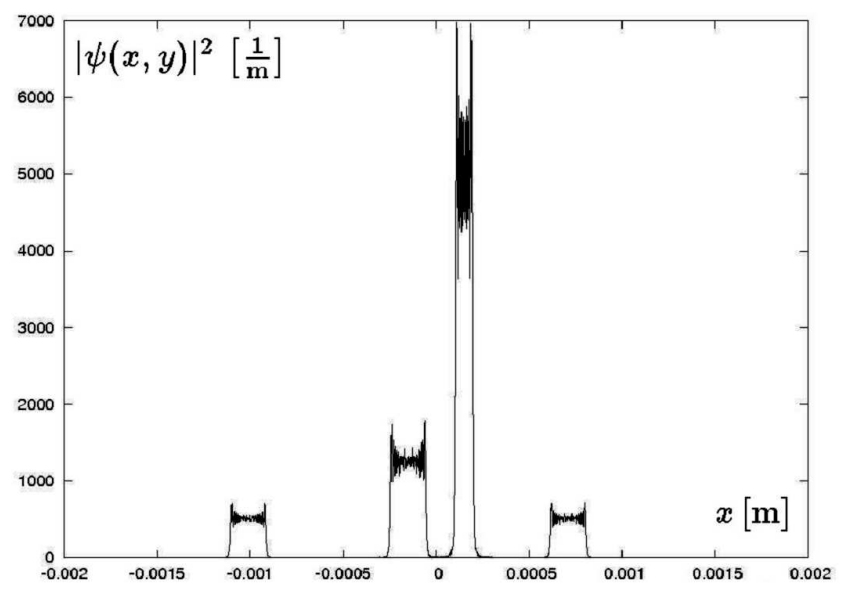

Fig. 2. Modulo square of a wave function at the distance $y=2 \mathrm{~m}$.

For the same complex grating modulo square of the transverse wave function at a distance $y=2 \mathrm{~m}$ is represented in Fig. 2. Central maxima associated with a slit and with a Ronchi grating are still separated. But at larger distances these two maxima overlap and the distribution of $x$ looks like the distribution of transverse momentum shown in Fig. 1.

\section{Probability of particle's arrivals to a screen}

Arsenović et al. [4] proposed a method to evaluate probability density of particle's coordinate $x$ at the distance $y=v t$ at time t using the probability density of particle's transverse momentum. Particle trajectories are approximated by straight lines. This approximation is very well justified in the far field, but not 
so well in the near field. It is argued that a particle with transverse momentum $p_{x}=\hbar k_{x}$, which was at a point $\left(x^{\prime}=x-\hbar k_{x} t / m, y=0\right)$, at time $t=0$ arrives to point $(x, y=v t)$ at time $t$. One has to integrate over all possible $k_{x}$ and $x^{\prime}$. Thus, the probability of particle's arrival to point $(x, y=v t)$ at time $t$ is

$$
P(x, y=v t)=\int_{-\infty}^{+\infty} \mathrm{d} k_{x} \int_{-\infty}^{+\infty} \mathrm{d} x^{\prime}\left|c\left(k_{x}\right)\right|^{2}\left|\psi\left(x^{\prime}, 0^{+}\right)\right|^{2} \delta\left(x-x^{\prime}-\hbar k_{x} t / m\right) .(6)
$$

From the above expression at a distance $y=2 \mathrm{~m}$ one finds the distribution represented in Fig. 3.

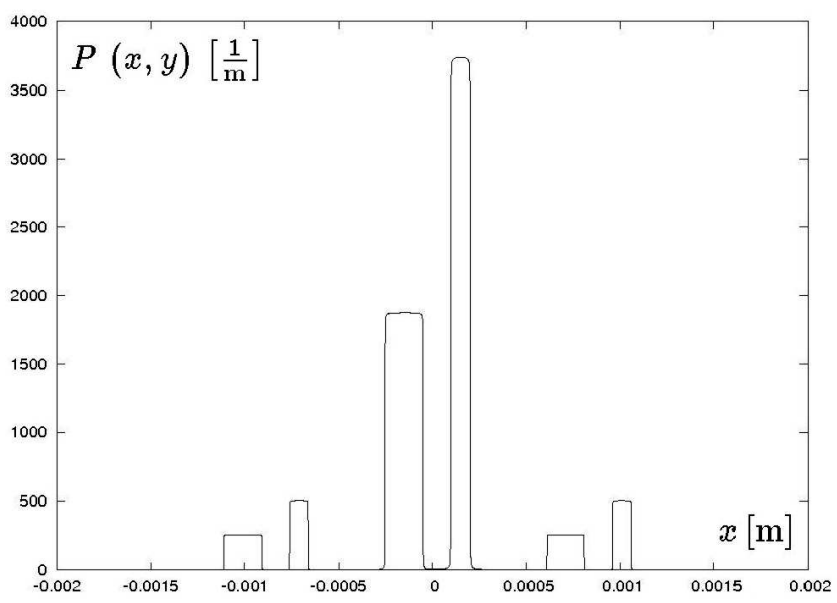

Fig. 3. Probability density of particles at a distance $y=2 \mathrm{~m}$ evaluated using Eq. (6).

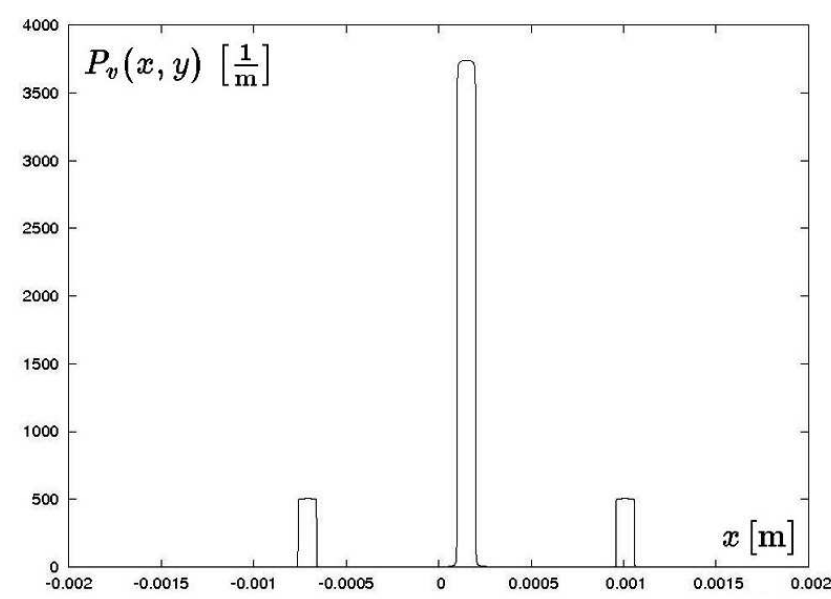

Fig. 4. Probability density at $y=2 \mathrm{~m}$ of particles passing through the wide slit A, only. 
There is a very good agreement with a distribution in Fig. 2, although to each small maximum in Fig. 2 corresponds a pair of small maxima in Fig. 3. The pairs are associated with small maxima of the transverse momentum distribution. But, one maximum of a pair is built by trajectories starting at slits of a Ronchi grating. Another maximum of a pair is built by trajectories starting at a wide slit.

To evaluate the distribution of particles which are so large that they may not pass through small slits of a Ronchi grating, but may pass through a wide slit, we apply the argumentation of Božić et al. [3]. The integration over $x^{\prime}$ in (6) is reduced to integration over wide slit A only. But, the distribution of transverse momentum of particles which pass through the wide slit remains the one showed in Fig. 1. It is why we predict that the distribution of large particles should be as shown in Fig. 4, that means it is influenced by the presence of the Ronchi grating, despite the fact that particles do not pass through this grating. But, the wave which is accompanying each particle passes through the wide slit, as well as through the small slits of a Ronchi grating.

\section{Conclusion}

It is shown that in an interference experiment with a beam of one per one Rydberg atom incident on a complex grating (consisting of one wide slit and a Ronchi grating) one could test the traditional assumption which reads: if a particle does not pass through a slit its accompanying wave does not pass either.

\section{References}

[1] C. Fabre, M. Gross, J.M. Raimond, S. Haroche, J. Phys. B 16, L671 (1983).

[2] M. Božić, D. Arsenović, L. Vušković, Bull. Am. Phys. Soc. 47, 74 (2002).

[3] M. Božić, D. Arsenović, L. Vušković, Phys. Rev. A 69, 053618 (2004).

[4] D. Arsenović, M. Božić, L. Vušković, J. Opt. B, Quantum Semiclass. Opt. 4, S358 (2002).

[5] M. Gondran, A. Gondran, Am. J. Phys. 73, 507 (2005).

[6] R. Feynman, A. Hibbs, Quantum Mechanics and Path Integrals, McGraw-Hill, New York 1965, p. 41.

[7] D. Arsenović, M. Božić, O.V. Manko, V.I. Manko, J. Russ. Laser Res. 26, 94 (2005). 